\title{
Can Dirac fermions destroy Yang-Mills black hole?
}

\author{
Łukasz Nakonieczny and Marek Rogatkd* \\ Institute of Physics \\ Maria Curie-Sklodowska University \\ 20-031 Lublin, pl. Marii Curie-Sklodowskiej 1, Poland
}

(Dated: July 16, 2018)

\begin{abstract}
We study the four-dimensional Einstein-Yang-Mills black hole in the presence of Dirac fermion field. Assuming a spherically symmetric static asymptotically flat black hole spacetime we consider both massless and massive fermion fields. The $(4+1)$-dimensional Einstein-Yang-Mills system effectively reducing to Einstein-Yang-Mills-Higgs-dilaton model, was also taken into account. One finds that fermion vacuum leads to the destruction of black holes in question.
\end{abstract}

PACS numbers: $04.50 .+\mathrm{h}$

\section{INTRODUCTION AND NOTATION.}

Gravitational collapse is one of the most important issue of general relativity and its extension to higher dimensional spacetime connected with $\mathrm{M} /$ string theory schemes of unification of the all known forces of Nature. One expects that a newly born black hole emerging from the gravitational collapse of a massive star will settle down to the stationary axisymmetric or static spacetime. The uniqueness theorem (or classification) of nonsingular black hole solutions states that a stationary axisymmetric solution of Einstein-Maxwell (EM) electrovacuum equations is isometrically diffeomorphic to the domain of outer communication of Kerr-Newman spacetime [1].

On the other hand, the complete classification of $n$-dimensional charged black holes both with non-degenerate and degenerate component of the event horizon was given in Refs. 22]. Partial results for the very nontrivial case of $n$ dimensional rotating black hole uniqueness theorem were provided in [3]. The aforementioned studies comprises also the case of extremal axisymmetric black hole both in EM theory and the low-energy limit of the string theory (the so-called EMAD-gravity) and supergravities theories [4].

As far as the uniqueness theorem of non-Abelian black holes is concerned, the situation is far more complicated (see [5] and references therein). It turned out that any static solution of Einstein-Yang-Mills (EYM) equations ought to either coincide with Schwarzschild one or posses some non-vanishing Yang-Mills (YM) charges. But it happened not to be the case when static black hole solutions with vanishing charges were discovered [6]. They were asymptotically indistinguishable from Schwarzschild black hole. Moreover, in Ref.[7] it was shown that static black hole of the considered class with magnetic charge needed not even be axially symmetric. In the light of the above, one can remark that the non-Abelian black holes reveal considerably more composed structure comparing to the EM ones.

Recently, studies of fermions in various backgrounds attract more attention. Exact solutions of the Dirac equation in curved spacetime may be a useful tool for investigations of physical properties of the considered spacetimes. Dirac fields were elaborated [10] in the context of EYM background found in Ref.[11], in the near-horizon limit of Kerr black hole 12], in Bertotti-Robinson spacetime [13], in spacetimes of black holes with nontrivial topology of the event horizon [14], in the vicinity of black holes with topological defects [15] and in the spacetime of black string [16]. The intermediate and late-time decays of massive Dirac fermions in various black hole spacetimes were also elaborated [28]-31.

The other tantalizing problem is the behaviour of black holes and the surrounding matter fields. Depending on the matter model in question, black holes may allow to exist the nontrivial fields outside the event horizon. Are there any configurations of matter fields that can destroy the emerging black hole? This question was tackled in Refs. [8]. In Refs. [9] it was revealed that Reissner-Nordström (RN) solution with both an electric and magnetic charges can be destroyed in the presence of a massless Dirac fermion field. On the other hand, it was revealed [17] that the only black hole solutions of four-dimensional spinor Einstein-dilaton-Yang-Mills field equations of motion were those for which spinors vanished identically outside black hole. It means that Dirac fermion fields either enter the black hole in question or escape to infinity. Recently, it was shown [19] that matter configuration composed of a perfect fluid could not be at rest outside a four-dimensional black hole in asymptotically flat static spacetime.

*Electronic address: rogat@kft.umcs.lublin.pl, marek.rogatko@poczta.umcs.lublin.pl, lnakonieczny@kft.umcs.lublin.pl 
In our paper we use the bosonozation technique by which the fermionic degrees of freedom can be described by a scalar field. We shall elaborate the problem of the influence of Dirac fermion fields on Yang-Mills (YM) black holes. In our considerations we assume that the black hole in question is spherically symmetric and static. We take into account the ordinary four-dimensional static spherically symmetric asymptotically flat EYM black hole and the fourdimensional Einstein-Yang-Mills-Higgs-dilaton (EYMHd) system deduced from five-dimensional EYM model while the Dirac fermion field will be treated in a s-wave sector.

The organization of this paper is as follows. In Sec.II we briefly review the basic facts concerning four-dimensional EYM black hole and Dirac fermion system. Then, we consider the massless as well as massive fermions case and their influence on black hole. Their asymptotical behaviours will be also discussed. Sec.III will be devoted to the backreaction process of Dirac fermions on YM fields. In Sec.IV we shall elaborate (4+1)-EYM system which effectively reduces to EYMd field equations, where both the matter fields and the line element coefficients do not depend on the fifth dimension. We conclude our investigations in Sec.V.

\section{FOUR-DIMENSIONAL EINSTEIN-YANG-MILLS BLACK HOLE AND DIRAC FERMIONS}

In this section we shall pay attention to static spherically symmetric solution of EYM field equations. In the case under consideration the line element can be provided by

$$
d s^{2}=-A^{2}(r) d t^{2}+B^{2}(r) d r^{2}+C^{2}(r) d \Omega^{2},
$$

where $d \Omega^{2}$ is a metric on $S^{2}$-sphere. In what follows it will be convenient to introduce the tortoise coordinate defined as $d r_{*}=\frac{B}{A} d r$. Just the underlying metric yields

$$
d s^{2}=-A^{2}(r) d t^{2}+A^{2}(r) d r_{*}^{2}+C^{2}(r) d \Omega^{2} .
$$

The main topic of our research will be the influence of Dirac fermion fields on EYM black hole. Fermions under consideration will be described by the Dirac equation provided by

$$
i \gamma^{\mu} D_{\mu} \psi-m \psi=0
$$

where the covariant derivative $D_{\mu}$ implies

$$
D_{\mu}=\nabla_{\mu}-i \lambda H_{\mu}
$$

$\lambda$ is the gauge coupling constant, while the component of Yang-Mills field have the forms as

$$
H_{\mu}=e_{\mu}^{i} H_{i}, \quad H_{i}=a_{i} n^{k} \tau_{k}+\frac{1-K(r)}{2 \lambda C} \epsilon_{i j k} n^{j} \tau^{k},
$$

where $a_{i}$ is the electric and $K$ the magnetic part of Yang-Mills vector. $n_{a}$ is the unit normal vector, while $\tau_{a}$ is a generator of $S U(2)$ group. On the other hand, $e_{\mu}^{i}$ are basis one-form defined by $g_{\mu \nu}=e_{\mu}^{i} e_{\nu}^{j} \eta_{i j}$, where $\eta_{i j}$ is metric tensor for Minkowski spacetime. The gamma Dirac matrices in a flat spacetime are defined by the relations

$$
\gamma^{0}=\left(\begin{array}{ll}
0 & 1 \\
1 & 0
\end{array}\right), \quad \gamma^{i}=\left(\begin{array}{cc}
0 & \sigma^{i} \\
-\sigma^{i} & 0
\end{array}\right) .
$$

where $\sigma^{i}$ are the usual Pauli matrices. It turned out that the Dirac operator takes the form as

$$
\gamma^{\mu} D_{\mu}=\left(\begin{array}{cc}
0 & D^{+} \\
D^{-} & 0
\end{array}\right)
$$

where by $D^{ \pm}$we have denoted the following relation:

$$
\begin{aligned}
D^{ \pm} & =A^{-1} \partial_{t}-i \lambda\left\{\left[\sigma^{0} a_{0} \pm \sigma^{1} a_{1}\right] \bar{n} \cdot \bar{\tau} \pm \frac{K-1}{2 \lambda C} \bar{n} \cdot \bar{\sigma} \times \bar{\tau}\right\}+ \\
& \pm \bar{\sigma} \cdot \bar{n} A^{-1} \partial_{r_{*}} \pm \bar{\sigma} \cdot \bar{n}\left\{A^{-1} C^{-1} \partial_{r_{*}} C+\frac{1}{2} A^{-1} A^{-1} \partial_{r_{*}} A\right\} \pm C^{-1} D_{S^{2}}
\end{aligned}
$$

Because of the fact that we restrict our attention to the $s$-wave sector, it can be spanned by two states $\chi_{1}$ and $\chi_{2}=\sigma^{a} n_{a} \chi_{1}$ being the hedgehog spinor ansatz [20]. Moreover, they will obey the properties as

$$
\begin{array}{rlr}
\bar{\sigma} \cdot \bar{n} \chi_{1 / 2} & =\chi_{2 / 1}, \quad D_{S^{2}} \chi_{1 / 2}=\mp \chi_{2 / 1}, \\
(\bar{n} \cdot \bar{\sigma} \times \bar{\tau}) \chi_{1 / 2}=\mp 2 i \chi_{2 / 1}, & \bar{n}(\bar{\sigma}+\bar{\tau}) \chi=0 .
\end{array}
$$


In terms of its components, spinor $\psi$ can be written as

$$
\psi=\left(\begin{array}{c}
\psi_{L} \\
\psi_{R}
\end{array}\right), \quad \psi_{L / R}=f_{L / R}\left(r_{r_{*}}, t\right) \chi_{1}+g_{L / R} \chi_{2} .
$$

By virtue of the above, the Lagrangian for two-component left and right-handed spinors implies

$$
\mathcal{L}_{F}=i \bar{\psi}_{R} D^{+} \psi_{R}+i \bar{\Psi}_{L} D^{-} \psi_{L}-m \bar{\psi}_{R} \psi_{L}-m \bar{\psi}_{L} \psi_{R}
$$

On this account we can integrate over the angular degrees of freedom in Dirac fermion action. From now on we will work with curved 2-dim spacetime: $d s^{2}=-A^{2} d t^{2}+A^{2} d r_{*}^{2}$. Latin letters from the beginning of alphabet will refer to the curved spacetime, i. e., $a=t, r_{*}$, while those from the end of the alphabet are bounded with the flat spacetime, i. e., $i=0,1$. In this spacetime the nonzero components of the spin connection is $\omega^{01}=\partial_{r_{*}} \ln A d t$, while the covariant derivative of spinor field has the form $\nabla_{a}=\partial_{a}+\frac{1}{2} \omega_{a}^{i j} \sigma^{i} \sigma^{j}$. In our considerations, we introduce two-dimensional spinors $F_{L / R}=\left(\begin{array}{c}f_{L / R} \\ g_{L / R}\end{array}\right)$ and we use the symmetric form of the Dirac operator $\gamma^{\mu} \stackrel{\leftrightarrow}{D}_{\mu}=\frac{1}{2}\left[\gamma^{\mu} \vec{D}_{\mu}-\gamma^{\mu} \overleftarrow{D}_{\mu}\right]$. It enables us to rewrite action for fermion fields in the form

$$
S_{F}=4 \pi \int d t \int A^{2} \mathcal{L}_{F}^{2} d r_{*}
$$

as well as the Lagrangian for them, which implies the following:

$$
\begin{array}{r}
\mathcal{L}_{F}^{(2)}=i C^{2} \bar{F}_{R} \stackrel{\leftrightarrow}{D^{+}}{ }_{\left(t, r_{*}\right)} F_{R}-C^{2} \frac{K}{C} \bar{F}_{R} \sigma^{2} F_{R}-C^{2} \lambda \bar{F}_{R}\left(\sigma^{i} a_{i}\right)^{+} \sigma^{1} F_{R}+i C^{2} A^{-1} \partial_{r_{*}} \ln (\sqrt{A} C) \bar{F}_{R} \sigma^{1} F_{R} \\
+i C^{2} \bar{F}_{L} \stackrel{\leftrightarrow}{D^{-}}{ }_{\left(t, r_{*}\right)} F_{L}+C^{2} \frac{K}{C} \bar{F}_{L} \sigma^{2} F_{L}-C^{2} \lambda \bar{F}_{L}\left(\sigma^{i} a_{i}\right)^{-} \sigma^{1} F_{L}-i C^{2} A^{-1} \partial_{r_{*}} \ln (\sqrt{A} C) \bar{F}_{L} \sigma^{1} F_{L} \\
-m C^{2} \bar{F}_{R} F_{L}-m C^{2} \bar{F}_{L} F_{R},
\end{array}
$$

where we have denoted by $D_{\left(t, r_{*}\right)}^{ \pm}=A^{-1}\left[\sigma^{0} \partial_{t} \pm \sigma^{1} \partial_{r_{*}}\right]$ and $\left(\sigma^{i} a_{i}\right)^{ \pm}=\sigma^{0} a_{0} \pm \sigma^{1} a_{1}$. We are now in a position to approach the question of the equations of motion for the above system. Namely, the Dirac equations for the two-dimensional fermions are provided by the relations

$$
\begin{gathered}
i D_{\left(t, r_{*}\right)}^{+} F_{R}-\frac{K}{C} \sigma^{2} F_{R}-\left(\lambda \sigma^{i} a_{i}\right)^{+} \sigma^{1} F_{R}+i A^{-1} \partial_{r_{*}} \ln (\sqrt{A} C) \sigma^{1} F_{R}+i \frac{1}{2 A C^{2}} \partial_{r_{*}} C^{2} \sigma^{1} F_{R}-m F_{L}=0 \\
i D_{\left(t, r_{*}\right)}^{-} F_{L}+\frac{K}{C} \sigma^{2} F_{L}-\left(\lambda \sigma^{i} a_{i}\right)^{-} \sigma^{1} F_{L}-i A^{-1} \partial_{r_{*}} \ln (\sqrt{A} C) \sigma^{1} F_{L}-i \frac{1}{2 A C^{2}} \partial_{r_{*}} C^{2} \sigma^{1} F_{L}-m F_{R}=0
\end{gathered}
$$

\section{A. Massless Dirac fermions}

In this subsection we shall focus our attention on massless right-handed spinors. One examines relation (15), which in two-dimensional spacetime $\left(t, r_{*}\right)$ may be rewritten as follows:

$$
i \sigma^{a} \nabla_{a} F_{R}-\lambda \sigma^{a} B_{a} \sigma^{1} F_{R}-V \sigma^{2} F_{R}+2 i \sigma^{r_{*}} \partial_{r_{*}} \ln C F_{R}=0,
$$

where we set $V=\frac{K}{C}$ and $B_{a}=e_{a}^{i} a_{i}$ being the electric part of Yang-Mills field. It can be readily find, by the direct computation, that the term $\sigma^{i} a_{i}$ now will be replaced by $\sigma^{a} B_{a}$. Further, in order to get rid of $\partial_{r_{*}} \ln C$ factor one can rescale spinors in the following way:

$$
G_{R} \equiv i \sigma^{3} e^{2 \int \partial_{r_{*}} \ln C d r_{*}} F_{R}
$$

It will be useful to choose the new basis for flat gamma matrices

$$
\begin{aligned}
\tilde{\gamma}^{0} & =-i \sigma^{3}, & \tilde{\gamma}^{1} & =-\sigma^{2}, \\
\left\{\tilde{\gamma}^{a}, \tilde{\gamma}^{b}\right\} & =2 \eta^{a b}, & \eta_{00} & =-1=-\eta_{11}, \\
\tilde{\gamma}^{3}=\tilde{\gamma}^{0} \tilde{\gamma}^{1} & =\sigma^{1}, & \tilde{\gamma}_{L / R} & =\frac{1}{2}\left(I \pm \tilde{\gamma}^{3}\right) .
\end{aligned}
$$

On this account, equations of motion yield

$$
i \tilde{\gamma}^{a} \nabla_{a} G_{R}+\lambda \tilde{\gamma}^{a} B_{a} \tilde{\gamma}^{3} G_{R}-V \tilde{\gamma}^{3} G_{R}=0 .
$$


It can be deduced that they may be derived from the Lagrangian of the form

$$
\mathcal{L}_{F R}=-i \bar{G}_{R} \tilde{\gamma}^{a} \nabla_{a} G_{R}-\lambda B_{a} \bar{G}_{R} \tilde{\gamma}^{a} \tilde{\gamma}^{3} G_{R}+V \bar{G}_{R} \tilde{\gamma}_{L} G_{R}-V \bar{G}_{R} \tilde{\gamma}_{R} G_{R} .
$$

It happened that $\mathcal{L}_{F R}$ can be examined by means of bosonization technique, i.e., the fermionic degrees of freedom can be described by a scalar field propagating in $\left(t, r_{*}\right)$-spacetime (see, e.g., [21]) One bosonizes the above Lagrangian by the following formulae

$$
\begin{aligned}
j_{R}^{a} \equiv \bar{G}_{R} \tilde{\gamma}^{a} G_{R} & =\frac{1}{\sqrt{\pi}} \varepsilon^{a b} \nabla_{b} \phi_{R}, \quad j_{3 R}^{a} \equiv \bar{G}_{R} \tilde{\gamma}^{a} \tilde{\gamma}^{3} G_{R}=\frac{1}{\sqrt{\pi}} \nabla^{a} \phi_{R}, \\
\bar{G}_{R} \tilde{\gamma}_{L} G_{R} & =b e^{2 i \sqrt{\pi} \phi_{R}}, \quad \bar{G}_{R} \tilde{\gamma}_{R} G_{R}=b^{*} e^{-2 i \sqrt{\pi} \phi_{R}},
\end{aligned}
$$

where $b$ and $b^{*}$ are constants depending on the normalization of the current $\bar{G} \gamma_{L / R} G$. For the brevity we set $b=b^{*}$. Thus the bosonized Lagrangian is provided by

$$
\mathcal{L}_{B R}=-\frac{1}{2} \nabla_{a} \phi_{R} \nabla^{a} \phi_{R}-\lambda B_{a} \frac{1}{\sqrt{\pi}} \nabla^{a} \phi_{R}+V b\left(e^{2 i \sqrt{\pi} \phi_{R}}-e^{-2 i \sqrt{\pi} \phi_{R}}\right),
$$

while equation of motion for $\phi_{R}$ yields

$$
\nabla_{a} \nabla^{a} \phi_{R}+\frac{\lambda}{\sqrt{\pi}} \nabla_{a} B^{a}+4 i b \sqrt{\pi} V \cos \left(2 \sqrt{\pi} \phi_{R}\right)=0 .
$$

Let us proceed to analyze the left-handed Dirac spinors. One can use the following substitution in order to get rid of $\partial_{r_{*}} \ln C$ term

$$
G_{L}=i \sigma^{3} e^{2 \int \partial_{r_{*}} \ln C d r_{*}} F_{L}
$$

We also choose the gamma matrices basis in the form as

$$
\tilde{\gamma}^{0}=-i \sigma^{3}, \quad \tilde{\gamma}^{1}=+\sigma^{2}, \quad \tilde{\gamma}^{3}=\tilde{\gamma}^{0} \tilde{\gamma}^{1}=-\sigma^{1} .
$$

Having all the above in mind the equation of motion for $G_{L}$-spinors implies

$$
i \tilde{\gamma}^{a} \nabla_{a} G_{L}-\lambda B_{a} \tilde{\gamma}^{a} \tilde{\gamma}^{3} G_{L}-V \tilde{\gamma}^{3} G_{L}=0 .
$$

On the other hand, the Lagrangian for $G_{L}$-spinors can be written as

$$
\mathcal{L}_{F L}=-i \bar{G}_{L} \tilde{\gamma}^{a} \nabla_{a} G_{L}+\lambda B_{a} \bar{G}_{L} \tilde{\gamma}^{a} \tilde{\gamma}^{3} G_{L}+V \bar{G}_{L} \tilde{\gamma}_{L} G_{L}-V \bar{G}_{L} \tilde{\gamma}_{R} G_{L}
$$

Replacing the fermionic degrees of freedom by the bosonization substitution given by the relations

$$
\begin{aligned}
j_{L}^{a} \equiv \bar{G}_{L} \tilde{\gamma}^{a} G_{L} & =\frac{1}{\sqrt{\pi}} \varepsilon^{a b} \nabla_{b} \phi_{L}, \quad j_{3 L}^{a} \equiv \bar{G}_{L} \tilde{\gamma}^{a} \tilde{\gamma}^{3} G_{L}=\frac{1}{\sqrt{\pi}} \nabla^{a} \phi_{L}, \\
\bar{G}_{L} \tilde{\gamma}_{L} G_{L} & =b e^{2 i \sqrt{\pi} \phi_{L}}, \quad \bar{G}_{L} \tilde{\gamma}_{R} G_{L}=b^{*} e^{-2 i \sqrt{\pi} \phi_{L}},
\end{aligned}
$$

we achieve the bosonized Lagrangian for the scalar fields

$$
\mathcal{L}_{B L}=-\frac{1}{2} \nabla_{a} \phi_{L} \nabla^{a} \phi_{L}+\lambda B_{a} \frac{1}{\sqrt{\pi}} \nabla^{a} \phi_{L}+V b\left(e^{2 i \sqrt{\pi} \phi_{L}}-e^{-2 i \sqrt{\pi} \phi_{L}}\right) .
$$

Accordingly, equation of motion implies the following:

$$
\nabla_{a} \nabla^{a} \phi_{L}-\frac{\lambda}{\sqrt{\pi}} \nabla_{a} B^{a}+4 b i \sqrt{\pi} V \cos \left(2 \sqrt{\pi} \phi_{L}\right)=0 .
$$

One can remark that the only difference between relations for $\phi_{L}$ and $\phi_{R}$ is the sign of the term containing $B_{a}$. However, in Ref. 22] it was pointed out that in order to have finite Yang-Mills black hole mass one needed to get $B_{a}=0$. By virtue of this argument we can readily verify that equations of motion for right and left-handed fermion fields are identical. Therefore, in what follows, we restrict our attention to only one equation of motion.

Having in mind the arguments quoted above, we commence with the asymptotic behaviour analysis of field $\phi_{R}$. From the point of view of demanding asymptotical flatness of the black hole solution in question, one has that 
$g_{t t} \sim g_{r r} \sim 1, r \sim r_{*}$ and $C=r$, as $r$-coordinate tends to infinity. It enables us to write the underlying equation of motion in the form as

$$
-\partial_{t}^{2} \phi_{R}+\partial_{r_{*}}^{2} \phi_{R}+4 i b \sqrt{\pi} \frac{K(\infty)}{r_{*}} \cos \left(2 \sqrt{\pi} \phi_{R}\right)=0
$$

where we set $V \sim \frac{K(\infty)}{r_{*}}, K(\infty)= \pm 1$. In order to satisfy our demands about finiteness of $\phi_{R}$ as $r_{*}$ tends to infinity the last term in relation (34) vanishes. In terms of it, we arrive at the equation

$$
-\partial_{t}^{2} \phi_{R}+\partial_{r_{*}}^{2} \phi=0
$$

with the regular solution provided by

$$
\phi_{R}=d e^{-i \omega\left(t \pm r_{*}\right)}
$$

where $d$ is an arbitrary constant. The obtained solution is time-dependent and admits the non-zero fermion current at infinity, i.e., $\nabla_{j} \phi_{R} \neq 0$.

Let us refine our studies to the near-horizon geometry of YM black hole surrounded by the Dirac fermion fields. In the vicinity of the event horizon one achieves

$$
A^{2}=N^{2} e^{\delta\left(r_{h}\right)}, \quad A^{2}=N^{2} e^{\delta\left(r_{h}\right)}, \quad N^{2}=2 \kappa\left(r-r_{h}\right),
$$

where $\kappa$ is the surface gravity and $e^{\delta\left(r_{h}\right)}$ is a constant factor.

Taking the usual change of variables $r-r_{h}=\rho^{-1}$, we obtain that

$$
r_{*}=-c \rho,
$$

where $c=\frac{1}{2 \kappa e^{\sqrt{\delta\left(r_{h}\right)}}}$. Exploring these relations we can rewrite equation of motion in the form as

$$
-\partial_{t}^{2} \phi_{R}+c^{2} \partial_{\rho}^{2} \phi_{R}+i \frac{8 b \sqrt{\pi} K\left(r_{h}\right) \kappa e^{\sqrt{\delta\left(r_{h}\right)}}}{r_{h} \rho} \cos \left(2 \sqrt{\pi} \phi_{R}\right)=0
$$

By the same reasoning as we followed in deriving equation (35), we conclude that dropping the last term in the above relation, the solution implies

$$
\phi_{R}=c_{1} e^{-i \omega\left(t \pm \frac{1}{c^{2}} \rho\right)}
$$

where we have set $c_{1}$ as integration constant.

Summing it all up, we conclude that the asymptotic analysis of scalar field equations being bosonized Dirac fermions, conducts to the time-dependent plane wave solutions. This is in contradiction to the static nature of the considered YM black hole. On this account, it is impossible to obtain static spherically symmetric YM black hole solution surrounded by Dirac fermion vacuum.

\section{B. Massive Dirac fermions.}

In this subsection we are mainly concerned with the Dirac massive case. In what follows we take into account the following ansatze for $F_{L}$ and $F_{R}$ :

$$
\begin{aligned}
& F_{L}=i \sigma^{3} F_{R} \equiv e^{-\int 2 \partial_{r_{*}} \ln C d r_{*}} G \\
& F_{R}=-i \sigma^{3} e^{-\int 2 \partial_{r_{*}} \ln C d r_{*}} G .
\end{aligned}
$$

Because of the fact that the left-handed fermions can be expressed in terms of a linear combination of the right-hand ones, we will use only the right-hand part of the original fermionic Lagrangian supplemented by the appropriate mass term. Namely, we obtain the equations of the form as

$$
i \sigma^{a} \nabla_{a} F_{R}-\frac{K}{C} \sigma^{2} F_{R}-\lambda \sigma^{a} B_{a} \sigma^{1} F_{R}+2 i A^{-1} \partial_{r_{*}} \ln (C) \sigma^{1} F_{R}-m i \sigma^{3} F_{R}=0 .
$$

On this account it is customary to write the following relations for $G$-fermions:

$$
i \tilde{\gamma}^{a} \nabla_{a} G-V \tilde{\gamma}^{3} G+\lambda \tilde{\gamma}^{a} B_{a} \tilde{\gamma}^{3} G-m G=0,
$$


where $\tilde{\gamma}$ are gamma matrices written in basis which is chosen like in the massless right-handed case. We also have that $V=\frac{K}{C}$. Hence, the effective Lagrangian for G-fermions will be provided by the expression

$$
\mathcal{L}_{G F}=-i \bar{G} \tilde{\gamma}^{a} \nabla_{a} G-\lambda B_{a} \bar{G} \tilde{\gamma}^{a} \tilde{\gamma}^{3} G+(V+m) \bar{G} \tilde{\gamma}_{L} G+(m-V) \bar{G} \tilde{\gamma}_{R} G .
$$

Next, as in the preceding sections, we try with the bosonization scheme

$$
\begin{aligned}
j^{a} \equiv \bar{G} \tilde{\gamma}^{a} G & =\frac{1}{\sqrt{\pi}} \varepsilon^{a b} \nabla_{b} \phi, \quad j_{3}^{a} \equiv \bar{G} \tilde{\gamma}^{a} \tilde{\gamma}^{3} G=\frac{1}{\sqrt{\pi}} \nabla^{a} \phi, \\
\bar{G} \tilde{\gamma}_{L} G & =b e^{2 i \sqrt{\pi} \phi}, \quad \bar{G} \tilde{\gamma}_{R} G=b^{*} e^{-2 i \sqrt{\pi} \phi} .
\end{aligned}
$$

When we set $b=b^{*}$, one enables to find Lagrangian for scalar field given by

$$
\mathcal{L}_{G B}=-\frac{1}{2} \nabla_{a} \phi \nabla^{a} \phi-\frac{\lambda}{\sqrt{\pi}} B_{a} \nabla^{a} \phi+(V+m) b e^{2 i \sqrt{\pi} \phi}+(m-V) b e^{-2 i \sqrt{\pi} \phi},
$$

where $b$ is constant. On the other hand, equation of motion for $\phi$ field implies

$$
\nabla_{a} \nabla^{a} \phi+\frac{\lambda}{\sqrt{\pi}} \nabla_{a} B^{a}+2 i b \sqrt{\pi}\left\{V\left[e^{2 i \sqrt{\pi} \phi}+e^{-2 i \sqrt{\pi} \phi}\right]+m\left[e^{2 i \sqrt{\pi} \phi}-e^{-2 i \sqrt{\pi} \phi}\right]\right\}=0,
$$

which can be rewritten in a more compact form

$$
\nabla_{a} \nabla^{a} \phi+\frac{\lambda}{\sqrt{\pi}} \nabla_{a} B^{a}+4 i b \sqrt{\pi}\{V \cos (2 \sqrt{\pi} \phi)+i m \sin (2 \sqrt{\pi} \phi)\}=0 .
$$

Applying the same analysis as in the preceding section lead us to the conclusion that in the near-horizon limit the bosonization field $\phi$ will be given in the form of a plane wave. Namely, it will be described by

$$
\phi=d_{1} e^{-i \omega\left(t \pm \frac{1}{c^{2}} \rho\right)},
$$

where $d_{1}$ is integration constant while $c$ is the same constant like in massless case.

On the other hand, in the limit when $r_{*} \rightarrow \infty$ the underlying equation of motion may be written as

$$
-\partial_{t}^{2} \phi+\partial_{r_{*}}^{2} \phi-4 b \sqrt{\pi} m \sin (2 \sqrt{\pi} \phi)=0,
$$

where due to the previously quoted arguments we have omitted $B_{a}$. Because of the fact that $V \sim 1 / r$, the term with cosine function tends to zero. Just we attain the form of the well known sine-Gordon equation, the solution of which implies

$$
\phi=\frac{2}{\sqrt{\pi}} \arctan \left(e^{-\sqrt{\frac{8 b \pi m}{1-v^{2}}}\left(r_{*}-v t\right)}\right)
$$

where $v$ is integration constant. From the above relation it can be inferred that fermion current tends to zero as $r_{*} \rightarrow \infty$.

To conclude this section, we remark that if we put $B_{a}=0$ we obtain the time-dependent Dirac fermions as well as through equations of motion, the time-dependent YM fields and the coefficients of the studied line element. All the above contradicts our primary assumptions about staticity of the the system in question.

\section{BACKREACTION ON YANG-MILLS FIELDS.}

If one considers a spherically symmetric spacetime being a Lorentz manifold on which $S O(3)$ group acts like isometry in such way that all group orbits are $S^{2}$-spheres, the spacetme in question may be locally written as a warped product of a two-dimensional Lorentz manifold and two-sphere with the standard metric on it [23]-[25]. It happened that it is convenient to rederive the field equations in the spherically symmetric case by varying the effective two-dimensional action. Putting in EYM action the ansatz for the YM gauge fields and the line element describing the symmetry in question, one can obtain the two-dimensional Lagrangian which yields

$$
\mathcal{L}_{Y M}=-\frac{C^{2}}{4} f_{a b} f^{a b}-|d K|^{2}-\frac{1}{2 C^{2}}\left(|K|^{2}-1\right)^{2},
$$


where the covariant derivative and the strength of $B_{a}$ are given by the relations

$$
d=\nabla_{a}-i B_{a}, \quad f_{a b}=\nabla_{a} B_{b}-\nabla_{b} B_{a} .
$$

We have denoted by a subscript $a=t, r_{*}$-coordinates, respectively. Equations of motion in the presence of the bosonized massless fermions are provided by

$$
\begin{aligned}
& \nabla_{a}\left[C^{2} f^{a b}\right]-2|K|^{2} B^{b}-\lambda j_{3 R}^{b}+\lambda j_{3 L}^{b}=0 \\
& \quad \nabla_{a} \nabla^{a} K-\frac{2}{C^{2}} K\left(|K|^{2}-1\right)+2 K B_{a} B^{a}+\frac{b}{C}\left[e^{2 i \sqrt{\pi} \phi_{R}}-e^{-2 i \sqrt{\pi} \phi_{R}}+e^{2 i \sqrt{\pi} \phi_{L}}-e^{-2 i \sqrt{\pi} \phi_{L}}\right]=0
\end{aligned}
$$

where $j_{3 R}^{a}=\bar{G}_{R} \tilde{\gamma}^{a} \tilde{\gamma}^{3} G_{R}$ and $j_{3 L}^{a}=\bar{G}_{L} \tilde{\gamma}^{a} \tilde{\gamma}^{3} G_{L}$. They can be reduced to the forms as follows:

$$
\begin{aligned}
& \nabla_{a}\left[C^{2} f^{a b}\right]-2|K|^{2} B^{b}-\frac{\lambda}{\sqrt{\pi}}\left[\nabla^{b} \phi_{R}-\nabla^{b} \phi_{L}\right]=0, \\
& \nabla_{a} \nabla^{a} K-\frac{2}{C^{2}} K\left(|K|^{2}-1\right)+2 K B_{a} B^{a}-i \frac{2 b}{C}\left[\sin \left(2 \sqrt{\pi} \phi_{R}\right)+\sin \left(2 \sqrt{\pi} \phi_{L}\right)\right]=0 .
\end{aligned}
$$

Having in mind equation (26) and (33), in the case when $B_{a}=0$, one can see that $j_{3 R}^{b}=j_{3 L}^{b}$. Thus, $B_{a}=0$ is the trivial solution of (54). As far as the influence fermions on YM fields is concerned, from relation (57) it can be inferred that the last term is not equal to zero and this leads to the conclusion that fermions have influenced on the magnetic part of YM fields. By virtue of the above, we observe that there is nonzero fermion contribution to magnetic part of Yang-Mills field. Since $\phi_{L}$ and $\phi_{R}$ are time dependent, then magnetic part of YM fields $K$ has also time dependence, which in turn cause that the coefficients of the line element in question also count on time.

In the case when $B_{a} \neq 0$, from equations (26) and (33), one can find fermion currents $j_{3 R}^{b}, j_{3 L}^{b}$. On the other hand, the inspection of equation (54) reveals the fact that fermion current causes the nontrivial solution for $B_{a}$. Consequently, black hole in question has both magnetic and electric charges. One gets the dyonic black hole. But this in turn leads to the infiniteness of the mass of the considered object [22].

As was remarked, taking into account massive fermions, one has that $F_{L}=i \sigma^{3} F_{R}$. This fact reduces the number of the independent fields, i.e., instead of $\phi_{L}$ and $\phi_{R}$ we have only one field $\phi$, satisfying the relation

$$
\nabla_{a}\left(C^{2} f^{a b}\right)-2|K|^{2} B^{b}-\frac{\lambda}{\sqrt{\pi}} \nabla^{b} \phi=0 .
$$

Hence, we get the black hole with both electric and magnetic charges (the dyon). On the other hand, it happened that this dyonic black hole can not have finite mass [22].

The above analysis reveals the fact that the presence of the Dirac fermion field leads to the destruction of a static YM black hole solution. First of all, the scalar which we get in the bosonization process are time-dependent. This in turn causes that magnetic part of YM fields as well as the coefficients of the line element in question depends also on time. It destroys are assumption about staticity of the considered black hole. These conclusions are true both for massless and massive Dirac fermions. Secondly, we can readily see that the presence of the Dirac fermion fields will provide the existence of the electric part of the YM fields, which in turn leads to the infiniteness of black hole mass.

\section{FIVE-DIMENSIONAL YANG-MILLS BLACK HOLE.}

It happens that studies of spherically symmetric solutions in higher dimensional theories reveals two ways of researches. One is connected with the assumption that we have spherically symmetric solutions in $n$-dimensions (this attitude is important in high-energy problems) and the other, when one assumes that solutions are spherically symmetric only in four-dimensional manifold. The second approach is importance from the point of view of the present Universe. These idea were explored in Refs. [26], where $(4+1)$-dimensional EYM systems were elaborated. It turned out that EYM system reduces to an effective four-dimensional EYMHd model. The $(4+n)$-dimensional case was considered in Ref.[27], with the assumption that all of the $n$-dimensional fields are independent on the extra dimensions. The solutions were spherically symmetric in four-dimensions while the additional dimensions were bounded with Ricci flat manifold.

Now, we shall proceed to elaborate $(4+1)$-dimensional EYM theory, where both matter fields and line element coefficients are independent on the fifth coordinate. Let us suppose that five-dimensional metric and five dimensional field are parameterized as follows:

$$
\begin{aligned}
{ }^{(5)} d s^{2}=g_{M N} d x^{N} d x^{M} & =e^{-\xi}\left[-A^{2} d t^{2}+B^{2} d r^{2}+C^{2} d \Omega^{2}\right]+e^{2 \xi}\left(d x^{5}\right)^{2}, \\
H_{M}^{a} d x^{M} & =H_{\mu}^{a} d x^{\mu}+\Phi^{a} d x^{5},
\end{aligned}
$$


where $M, N=t, r, \theta, \phi, x^{5}, a$ is a group index, $H_{M}$ is the five-dimensional Yang-Mills field. On the other hand, $H_{\mu}$ denotes the four-dimensional Yang-Mills field components and $\xi$ plays the role of the dilaton [26]. The above relations provide that we attain to the following effective four-dimensional EYMD theory for which the Lagrangian after compactification yields

$$
\mathcal{L}_{4}=a_{1} R-a_{2} \nabla_{\mu} \xi \nabla^{\mu} \xi-\frac{1}{4} e^{\xi} F_{\mu \nu}^{a} F^{a \mu \nu}-\frac{1}{2} e^{-2 \xi} D_{\mu} \Phi^{a} D^{\mu} \Phi^{a}
$$

where $a_{1}$ and $a_{2}$ are constants depending on five dimensional gravitational constant while the covariant derivative of the Higgs field in the adjoint representation implies

$$
D_{\mu} \Phi^{a}=\nabla_{\mu} \Phi^{a}+\varepsilon_{a b c} H_{\mu}^{b} \Phi^{c}
$$

Let us assume further, that $\Phi=\nu Y(r) \tau^{i} n_{i}$, where $\nu$ is the expectation value of the Higgs field.

The matter Lagrangian written in $\left(t, r_{*}\right)$-coordinates is given by

$$
\mathcal{L}_{2-d i m}=-a_{2} C^{2} \nabla_{a} \xi \nabla^{a} \xi-\frac{C^{2}}{4} e^{\xi} f_{a b} f^{a b}-e^{\xi}(d K)^{2}-\frac{1}{2 C^{2}} e^{\xi}\left(|K|^{2}-1\right)^{2}-\frac{C^{2}}{2} e^{-2 \xi} \nabla_{a} Y \nabla^{a} Y-e^{-2 \xi}|K|^{2} Y^{2}
$$

As far as the five-dimensional fermions is concerned, after dimensional reduction their action yields

$$
S_{F_{4}}=\int \sqrt{-{ }^{(4)} g} d^{4} x\left[i \bar{\psi} \gamma^{\mu} \stackrel{\leftrightarrow}{D}_{\mu} \psi+\lambda_{2} \bar{\psi} \gamma^{5} e^{-2 \xi} \Phi^{i} \tau^{i} \psi\right]
$$

where $\gamma^{5}=\left(\begin{array}{cc}I & 0 \\ 0 & -I\end{array}\right)$. One can observe that they gain mass by coupling to the five-dimensional component of YM field $\Phi^{i}$. Namely it implies

$$
-m\left[\bar{\psi}_{R} \psi_{L}+\bar{\psi}_{L} \psi_{R}\right] \rightarrow \lambda_{2} e^{-2 \xi} \bar{\psi}_{R} \Phi^{i} \tau^{i} \psi_{L}-\lambda_{2} e^{-2 \xi} \bar{\psi}_{L} \Phi^{i} \tau^{i} \psi_{R},
$$

where $\lambda_{2}$ is fermion coupling constant and $\tau^{i}$ are $S U(2)$ generators. On the other hand, $\Phi^{i}$ resembles Higgs fields in four-dimensional background. Massless fermions will be described by the same equations and therefore we restrict our attention to the massive case.

Further, we represent $\psi_{L / R}$ in terms of $\chi_{1 / 2}$

$$
\lambda_{2} e^{-2 \xi} \bar{\psi}_{R} \Phi^{i} \tau^{i} \psi_{L}=-C^{2} \lambda_{2} \nu Y e^{-2 \xi} \bar{F}_{R} \sigma^{1} F_{L}
$$

Expressing fermions as two-dimensional ones and integrating over the angles, we get the effective two-dimensional Lagrangian given by (14) with following substitutions:

$$
\begin{aligned}
& -m \bar{F}_{R} F_{L} \rightarrow-\lambda_{2} \nu e^{-2 \xi} Y \bar{F}_{R} \sigma^{1} F_{L}, \\
& -m \bar{F}_{L} F_{R} \rightarrow \lambda_{2} \nu e^{-2 \xi} Y \bar{F}_{L} \sigma^{1} F_{R} .
\end{aligned}
$$

Bosonization of the Dirac fermion fields will take place as in the preceding sections. Namely, we introduce $G$-fermions $F_{L}=i \sigma^{3} F_{R}$ and $F_{R}=-i \sigma^{3} e^{-\int 2 \partial_{r_{*}} \ln (C) d r_{*}} G$, where two-dimensional base of the gamma matrices is chosen as follows:

$$
\tilde{\gamma}^{0}=-i \sigma^{3}, \quad \tilde{\gamma}^{1}=-\sigma^{2}, \quad \tilde{\gamma}^{3}=\tilde{\gamma}^{0} \tilde{\gamma}^{1}
$$

It leads us to the equation of motion of the form as

$$
i \tilde{\gamma}^{a} \nabla_{a} G-V^{\prime} \tilde{\gamma}^{3} G+\lambda \tilde{\gamma}^{a} B_{a} \tilde{\gamma}^{3} G=0,
$$

where we set $V^{\prime}=\frac{K}{C}+\lambda_{2} \nu e^{-2 \xi} Y$. They can be derived from the effective Lagrangian provided by

$$
\mathcal{L}_{G F}=-i \bar{G} \tilde{\gamma}^{a} \nabla_{a} G-\lambda \bar{G} \tilde{\gamma}^{a} B_{a} \tilde{\gamma}^{3} G+V^{\prime} \bar{G} \tilde{\gamma}_{L} G-V^{\prime} \bar{G} \tilde{\gamma}_{R} G .
$$

It can be easily verified that using following bosonization formulae

$$
\begin{aligned}
j^{a}=\bar{G} \tilde{\gamma}^{a} G & =\frac{1}{\sqrt{\pi}} \varepsilon^{a b} \nabla_{b} \phi, \quad j_{3}^{a}=\bar{G} \tilde{\gamma}^{a} \tilde{\gamma}^{3} G=\frac{1}{\sqrt{\pi}} \nabla^{a} \phi \\
\bar{G} \tilde{\gamma}_{L} G & =b e^{2 i \sqrt{\pi} \phi}, \quad \bar{G} \tilde{\gamma}_{R} G=b^{*} e^{-2 i \sqrt{\pi} \phi}
\end{aligned}
$$


and setting $b=b^{*}$, the bosonized Lagrangian becomes

$$
\mathcal{L}_{G B}=-\frac{1}{2} \nabla_{a} \phi \nabla^{a} \phi-\frac{\lambda}{\sqrt{\pi}} B_{a} \nabla^{a} \phi+V^{\prime} b\left[e^{2 i \sqrt{\pi} \phi}-e^{-2 i \sqrt{\pi} \phi}\right] .
$$

Consequently, equation of motion for scalar field $\phi$ is given by

$$
\nabla_{a} \nabla^{a} \phi+\frac{\lambda}{\sqrt{\pi}} \nabla_{a} B^{a}+2 i b \sqrt{\pi} V^{\prime}\left[e^{2 i \sqrt{\pi} \phi}+e^{-2 i \sqrt{\pi} \phi}\right]=0,
$$

or in a more compact form it implies

$$
\nabla_{a} \nabla^{a} \phi+\frac{\lambda}{\sqrt{\pi}} \nabla_{a} B^{a}+4 i b \sqrt{\pi} V^{\prime} \cos (2 \sqrt{\pi} \phi)=0 .
$$

\section{Asymptotic analysis of equations of motions.}

Taking the same change of variable like in massless case, namely $r_{*} \sim-\rho$, and noticing that $A^{2} \sim \rho^{-1}$ we see that in near-horizon limit $\phi$ is given by the plane wave solution. For the finiteness of black hole mass we assume that $B_{a}=0$, then one arrives at

$$
\phi=e_{1} e^{-i \omega\left(t \pm \frac{1}{c^{2}} \rho\right)},
$$

where $e_{1}$ is arbitrary constant while $c^{2}$ is the same like in the previously studied massless case. Thus, if we demand $B_{a}=0$, the regular solution for $\phi$ must be time-dependent.

Now we will look for solution in $r_{*} \rightarrow \infty$ limit. Namely, one has that

$$
-\partial_{t}^{2} \phi+\partial_{r_{*}}^{2} \phi+4 i b \sqrt{\pi} m_{0} \cos (2 \sqrt{\pi} \phi)=0,
$$

where $m_{0}=\lambda_{2} \nu Y_{0} e^{-2 \xi_{0}}, Y_{0}$ and $\xi_{0}$ are the asymptotic values of Higgs and dilaton fields, respectively. One can rewriting $\phi$ as $\phi\left(r_{*}\right)=\phi_{a}\left(r_{*}\right)-\frac{1}{2 \sqrt{\pi}} \frac{\pi}{2}$, which leads us to the following form of equations of motion

$$
-\partial_{t}^{2} \phi_{a}+\partial_{r_{*}}^{2} \phi_{a}-4 i b \sqrt{\pi} m_{0} \sin \left(2 \sqrt{\pi} \phi_{a}\right)=0 .
$$

As we see it is sine-Gordon equation with complex coefficients. Its solution yields

$$
\phi_{a}=\frac{2}{\sqrt{\pi}} \arctan \left(e^{-\sqrt{\frac{8 i \pi b m_{0}}{1-v^{2}}}\left(r_{*}-v t\right)}\right),
$$

where $\nu$ is an integration constant.

It can be remarked that $\phi$ tends to the constant value $-\frac{\sqrt{\pi}}{4}$ as $r_{*}$ goes to infinity. But $\phi$ itself is not a physical quantity. Physical quantities are fermions fields and they are given by derivatives of $\phi$. Concluding, in both cases of massive fermions (normal and Higgs generated mass) fermionic currents decay at infinity.

The analysis of fermion backreaction on Yang-Mills field goes along the same line like in pure four-dimensional case. Conclusion are qualitatively the same. Namely, time-dependent fermion field leads to the destruction of static ansatz for Yang-Mills black hole (massive and massless case), or massive fermions lead to the appearance of the nonzero electric part of YM field.

\section{CONCLUSIONS}

In our paper we have considered the influence of the Dirac fermion field on EYM black hole. One takes into account two cases, i.e., the four-dimensional YM black hole [22] and black hole in five-dimensional generalization of YM theory. In the latter case the five-dimensional theory reduces to the four-dimensional EYMHd model. In both cases we elaborated $S U(2)$ YM theory and treated Dirac fermion in s-wave sector. Assuming a spherically symmetric static asymptotically flat black hole spacetime, we bosonize fermion fields and study equations of motion for the obtained scalar fields.

In a massless fermion sector we arrive at two scalar fields $\phi_{L}$ and $\phi_{R}$ corresponding respectively to the left and right-handed Dirac fermions. It happened that the action governing the scalar fields differs only by the sign in the 
term connected with electric part of YM field. Because of the fact that the finite mass YM black hole configuration exists only when electric part of YM field is equal to zero, the resulting equations of motion for both scalar fields are identical. The analysis of $\phi_{L / R}$ fields in the near-horizon and near infinity limits, reveals the fact that they are given by plane wave solutions. It in turns leads to the time-dependence of magnetic part of YM field as well as through EYM field equations to the time-dependence of the considered line element coefficients.

The situation is slightly different in massive fermions case. First of all one should recognize two kinds of mass terms emerging in our considerations. Namely, the ordinary mass term $m \bar{\psi} \psi$ appears in four-dimensional spacetime and the mass term connected with Higgs field $\lambda \Phi \bar{\psi} \psi$ in five-dimensional case which reduces effectively to the four-dimensional EYMHd theory. Before bosonization we use simplifying assumption that the right and left-handed part of the Dirac fermion field are connected through the transformation $F_{R}=i \sigma^{3} F_{L}$. It allows us to express Dirac fermion field by only one scalar field $\phi$. Next step was to analyze the beahaviour of $\phi$ filed in the near-horizon and infinity limits. It turns out that in the near-horizon limit the solution is described by a plane wave. This conclusion is true for both aforementioned masses. On the other hand, as far as the near infinity limit is concerned, equations of motion for ordinary mass term $m \bar{\psi} \psi$ reduces to the sine-Gordon type of equation. This equation has a time-dependent decaying solution as one approaches near infinity limit (the so-called anti-kink solution). In the case of the Higgs generated mass term $\lambda \Phi \bar{\psi} \psi$, equations in question can be also brought to the sine-Gordon type of equation but with a complex coefficient. It can be found that the solution reduces to the decaying oscillation function plus a nonzero constant term. Moreover, the presence of the Dirac fermion field currents will cause non-trivial value of the electric part of the YM fields, which in turn leads to the infiniteness of black hole mass.

Summing it all up, we remark that the presence of Dirac fermion field (fermion vacuum) in the spacetime of magnetically charged spherically symmetric static asymptotically flat YM black hole will lead to the destruction of this black object. It will happen by the destroying the static ansatz for black hole by both massive and massless Dirac fermions. Asymptotical analysis of the behaviour of the fermion fields suggests that the massless fermions are propagating in the whole spacetime, while massive Dirac fermions are confined to the near-horizon region. This conclusion remains true for the effectively reduced five-dimensional YM theory. Just, in order to have static YM black hole one should have Dirac fermions which are constant in the domain of outer communication of the black hole in question or Dirac fermion fields ought to enter the black hole (massive Dirac field) or escape to infinity (massless Dirac fermions). This is just the same conclusion as conceived in Refs. [17].

\section{Acknowledgments}

Ł N was supported by Human Capital Programme of European Social Fund sponsored by European Union. MR was partially supported by the grant of the National Science Center 2011/01/B/ST2/00408.

[1] M.Heusler, Black Holes Uniqueness Theorems (Cambridge University Press, Cambridge, England, 1996).

[2] Gibbons G W, Ida D and Shiromizu T 2002 Phys. Rev. D 66, 044010 (,)

G.W.Gibbons, D.Ida, and T.Shiromizu, Phys. Rev. Lett. 89, 041101 (2002),

S.Hollands, A.Ishibashi, and R.M.Wald, Commun. Math. Phys. 271, 699 (2007),

M.Rogatko, Class. Quantum Grav. 19, L151 (2002),

M.Rogatko, Phys. Rev. D 67, 084025 (2003),

M.Rogatko, ibid. 70, 084025 (2004),

M.Rogatko, ibid. 73, 124027 (2006).

[3] Y.Morisawa and D.Ida, Phys. Rev. D 69, 124005 (2004),

Y.Morisawa, S.Tomizawa, and Y.Yasui, Phys. Rev. D 77, 064019 (2008),

M.Rogatko, Phys. Rev. D 77, 124037 (2008),

S.Hollands and S.Yazadjiev, Commun. Math. Phys. 283, 749 (2008), S.Hollands and S.Yazadjiev, Class. Quantum Grav. 25, 095010 (2008),

D.Ida, A.Ishibashi, and T.Shiromizu, Prog. Theor. Phys. Suppl. 189, 52 (2011).

[4] A.K.M.Massod-ul-Alam, Class. Quantum Grav. 14, 2649 (1993),

M.Mars and W.Simon, Adv. Theor. Math. Phys. 6, 279 (2003),

M.Rogatko, Class. Quantum Grav. 14, 2425 (1997),

M.Rogatko, Phys. Rev. D 58, 044011 (1998),

M.Rogatko, ibid. 59, 104010 (1999),

M.Rogatko, ibid. 82, 044017 (2010),

M.Rogatko, Class. Quantum Grav. 19, 875 (2002),

S.Tomizawa, Y.Yasui, and A.Ishibashi, Phys. Rev. D 79, 124023 (2009), 
S.Tomizawa, Y.Yasui, and A.Ishibashi, Phys. Rev. D 81, 084037 (2010), J.B.Gutowski, JHEP 0408, 049 (2004),

J.P.Gauntlett, J.B.Gutowski, C.M.Hull, S.Pakis, and H.S.Real, Class. Quantum Grav. 20, 4587 (2003).

[5] M.Heusler, Hell. Phys. Acta 69, 501 (1996),

P.Bizon, Acta Phys. Polonica B 25, 877 (1994).

[6] H.P.Künzle, A.K.M.Masood-ul-Alam, J. Math. Phys. 31, 928 (1990),

P.Bizon, Phys. Rev. Lett. 64, 2844 (1990),

M.S.Volkov and D.V.Galtsov, JETP Lett. 50, 346 (1989).

[7] S.A.Ridgway and E.J.Weinberg, Phys. Rev. D 52, 3440 (1995).

[8] P.Hajicek, J. Math. Phys. 15, 1554 (1974),

G.W.Gibbons, Phys. Rev. D 15, 3530 (1974).

[9] D.Lohiya, Phys. Rev. D 30, 1194 (1984).

[10] G.W.Gibbons and A.R.Steif, Phys. Lett. B 314, 13 (1993).

[11] R.Bartnik and J.McKinnon, Phys. Rev. Lett. 61, 141 (1988).

[12] I.Sakalli and M.Halilsoy, Phys. Rev. D 69, 124012 (2004).

[13] G.Silva-Ortigoza, Gen. Rel. Grav. 33, 395 (2001), I.Sakalli, ibid. 35, 1321 (2003).

[14] M.Góźdź, L.Nakonieczny, and M.Rogatko, Phys. Rev. D 81, 104027 (2010), M.Góźdź and M.Rogatko, Int. J. Mod. Phys. E 20, 507 (2011).

[15] L.Nakonieczny and M.Rogatko, Phys. Rev. D 84, 044029 (2011),

[16] L.Nakonieczny and M.Rogatko, Phys. Rev. D 85, 044013 (2012).

[17] F.Finster, J.Smoller, and S.T.Yau, Adv. Theor. Math. Phys. 4, 1231 (2000).

[18] F.Finster, J.Smoller, and S.T.Yau, Nucl. Phys. B 584, 387 (2000), F.Finster, J.Smoller, and S.T.Yau, Mich. Math. j. 47, 199 (2000), F.Finster, J.Smoller, and S.T.Yau, Commun. Math. Phys. 205, 249 (1999), F.Finster, J.Smoller, and S.T.Yau, J. Math. Phys. 41, 2173 (2000).

[19] T.Shiromizu and S.Yamada, J. Math. Phys. 47, 112502 (2006).

[20] M.Kim and M.K.Banerjee, Phys. Rev. C 48, 2035 (1993), A.Chodos and C.B.Thorn, Phys. Rev. D 12, 2733 (1975).

[21] S.Coleman, Phys. Rev. D 11, 2088 (1981),

R.E.Gamboa Saravi, F.A.Schaposnik, and H.Vucetich, ibid. 30, 363 (1984), J.Bartelos-Neto and A.Das, ibid. 33, 2262 (1986), C.P.Burgess and F.Quevedo, Nucl. Phys. B 421, 373 (1999).

[22] P.Bizon and O.Popp, Class. Quantum Grav. 9, 193 (1992).

[23] M.S.Volkov and D.V.Galtsov, Phys. Reports 319, 1 (1999).

[24] O.Brodbeck, M.Heusler, and N.Straumann, Phys. Rev. D 53, 754 (1996).

[25] P.Cordero and C.Teitelboim, Ann. Phys. 100, 607 (1976).

[26] M.S.Volkov, Phys. Lett. B 524, 369 (2002), Y.Brihaye and B.Hartmann, ibid. 534, 137 (2002), B.Hartmann, ibid. 602, 231 (2004).

[27] Y.Brihaye, F.Clement, and B.Hartmann, Phys. Rev. D 70, 084003 (2004).

[28] J.L.Jing, Phys. Rev. D 70, 065004 (2004).

[29] J.L.Jing, Phys. Rev. D 72, 027501 (2005).

[30] G.W.Gibbons and M.Rogatko, Phys. Rev. D 77, 044034 (2008).

[31] G.W.Gibbons, M.Rogatko, and A.Szyplowska, Phys. Rev. D 77, 064024 (2008), M.Rogatko and A.Szyplowska, Phys. Rev. D 79, 104005 (2009). 